\title{
Strates
}

STRATES Matériaux pour la recherche en sciences sociales

$12 \mid 2006$

Nouvelles tensions impériales et recompositions en Europe centrale, orientale, et CEI

\section{Sibérie extrême-orientale : nature et ville post- communiste en république Sakha (Iakoutie)}

Émilie MAJ

\section{(2) OpenEdition \\ Journals}

Édition électronique

URL : http://journals.openedition.org/strates/2392

ISSN : $1777-5442$

Éditeur

Laboratoire Ladyss

Édition imprimée

Date de publication : 31 décembre 2006

ISSN : 0768-8067

\section{Référence électronique}

Émilie MAJ, «Sibérie extrême-orientale : nature et ville post-communiste en république Sakha

(lakoutie) », Strates [En ligne], 12 | 2006, mis en ligne le 26 juillet 2007, consulté le 07 septembre 2020. URL : http://journals.openedition.org/strates/2392

Ce document a été généré automatiquement le 7 septembre 2020

Tous droits réservés 


\title{
Sibérie extrême-orientale : nature et ville post-communiste en république Sakha (Iakoutie)
}

\author{
Émilie MAJ
}

1 La Iakoutie ${ }^{1}$, connue des Russes depuis leur arrivée en 1932 où ils fondèrent la ville de Iakoutsk, est entrée dans la sphère soviétique en 1918 sous le nom de République autonome socialiste soviétique de Iakoutie (IaASSR). En 1990, après la chute du bloc communiste, elle a adopté le statut de république souveraine et porte à présent le nom de République sakha² (Iakoutie) [RS(Ia)]. Le statut de souveraineté a été aboli par Vladimir Poutine depuis les dernières élections présidentielles. Durant les années soviétiques, la République autonome, dotée de territoires considérables et d'un faible taux de population, a été le siège de grands travaux de construction et d'exploitation des ressources naturelles. La densité de population, inférieure à 1 habitant par $\mathrm{km}^{2}$, a fait d'elle une plate-forme d'essais atomiques à l'image du Kazakhstan.

2 Les communistes ont été, dans certaines Républiques, les fondateurs des villes modernes. Au sud, en république Touva, ils ont fixé la population nomade dans des villages créés de toutes pièces. En Iakoutie, si les villages iakoutes existaient au moins depuis l'arrivée des Russes, autrement dit depuis le xviI ${ }^{\mathrm{e}}$ siècle, il est certain que la sédentarisation de nombreux nomades a continué avec la politique de collectivisation des années 1920-1930 et que les agglomérations de taille conséquente créées au $\mathrm{xx}^{\mathrm{e}}$ siècle eurent pour vocation première d'être des cités minières. En voulant changer la société et la "développer ", le communisme provoqua une évolution des mentalités qui engendra une perte des valeurs écologiques des ancêtres autochtones qui vivaient d'élevage, de chasse et de pêche sur le territoire de la Iakoutie. D'après les statistiques de 1996, la population actuelle de cette République est répartie entre une moitié de Russes et une autre composée par les minorités de Sibérie, dont les Iakoutes forment la part la plus importante, soit $39 \%$ de Iakoutes, $47 \%$ de Russes, $6 \%$ d'Ukrainiens, 1,5\% de Biélorusses, 1,5\% d'Evenks, $1 \%$ d'Evènes, $0,07 \%$ de Ioukaguirs et $0,05 \%$ de Tatars ( $c f$. tableau 1). Malgré le recul des valeurs liées au respect de la nature, l'expression « nous 
sommes les enfants de la nature" fait largement partie à la fois des expressions employées par les habitants autochtones non slaves et des slogans et discours politiques. Ceci nous conduit à nous interroger sur la relation entretenue aujourd'hui vis-à-vis de la nature dans cette partie lointaine de l'ex-bloc communiste qui a mis, dans ses priorités, jusqu'à une période récente, l'application d'une politique de protection de la nature pour faire face aux dégâts considérables qui ont été commis à l'égard de l'environnement naturel sibérien lors de la période soviétique et qui mettent gravement en danger la santé des habitants.

On ne considèrera pas tant ici les mentalités des autochtones, leur passé animiste et la perte de leurs valeurs anciennes de conservation de la nature, que l'espace citadin réel, resitué dans la sphère économique, sociale et écologique. Qu'est-ce aujourd'hui qu'une ville dans un pays de taïga et de toundra qui vit essentiellement de l'industrie minière? Comment l'espace citadin s'inscrit-il dans l'environnement? Quelles relations entretient-il à la nature ? Pour répondre à ces questions, nous décrirons tout d'abord l'espace environnemental de la nature, des campagnes et des villes. Puis, nous consacrerons une autre partie à l'importance de ces relations dans la politique écologique en République sakha (Iakoutie).

\begin{tabular}{|c|c|c|c|c|c|}
\hline & & Population citadine & & Population villageoise & \\
\hline Population totale & 718332 & totale & 481784 & totale & 236548 \\
\hline lakoutes & 310182 & lakoutes & 115027 & lakoutes & 195155 \\
\hline Russes & 306567 & Russes & 285968 & Russes & 20599 \\
\hline Ukrainiens & 31062 & Ukrainiens & 29470 & Ukrainiens & 1592 \\
\hline Evenks & 12487 & Evenks & 3196 & Evenks & 9291 \\
\hline Evènes & 8083 & Evènes & 2712 & Evènes & 5371 \\
\hline Tatars & 9049 & Tatars & 8353 & Tatars & 696 \\
\hline Bouriates & 5648 & Bouriates & 5261 & Bouriates & 387 \\
\hline Dolganes & 771 & Dolganes & 125 & Dolganes & 646 \\
\hline lakoukaguirs & 741 & lakoukaguirs & 313 & lakoukaguirs & 428 \\
\hline
\end{tabular}

Tableau 1. Nombre d'habitants et répartition de la population de la République sakha par nationalités selon le recensement de la Fédération russe de 2002.

Villes industrielles, cités ouvrières et villages agricoles

4 D'après le recensement de 1993, la Iakoutie occupe une surface de $3103200 \mathrm{~km}^{2}$ pour 1086500 habitants ( $C f$ en document annexe: carte 1. Types d'agglomération en lakoutie). Une grande partie de la population se concentre en Iakoutie centrale et dans 
le sud. En effet, c'est en Iakoutie centrale que se trouve Iakoutsk, la capitale où vivent 200000 personnes, avec deux centres importants: les villes de Namtsy au nord et de Pokrovsk au sud. Au sud de la Iakoutie, 80000 personnes se concentrent dans la ville industrielle de Nerungri. À l'ouest, Mirny ${ }^{3}$, importante zone d'extraction de diamants, concentre une population de 80000 personnes, périphérie comprise. La Iakoutie comporte en tout treize agglomérations possédant le statut de "villes", dont font partie Iakoutsk, Mirny, Udatchny, Viliuï, Niurba, Olekminsk, Nerungri ainsi que Verkhoïansk (cf. tableau 2). Cette dernière est située au-dessus du cercle polaire et n'a de ville que le statut officiel : paradoxe de la Iakoutie, Verkhoïansk ressemble plus à un village, avec une population d'à peine quelques milliers d'âmes, des maisons en bois de plein pieds ou à un étage et une des températures les plus basses de tout l'hémisphère nord ${ }^{4}$. En terme d'importance de population, les villes sont suivies par les cités minières qui sont au nombre de 60 et les villages agricoles qui vivent essentiellement des activités d'élevage de bétail (bovins et chevaux), situés dans des zones de prairie souvent désignées en iakoute par le terme alaas ${ }^{5}$. On en dénombre 559, dispersés dans les 33 oulous ${ }^{6}$ de Iakoutie.

\begin{tabular}{|l||l|l|l|l|l|l|l|l|}
\hline & $\begin{array}{l}\text { superficie } \\
\text { du } \\
\text { territoire } \\
\text { par } \\
\text { milliers } \\
\text { de km }\end{array}$ & régions & villes & $\begin{array}{l}\text { agglomérations } \\
\text { de type citadin }\end{array}$ & $\begin{array}{l}\text { administrations } \\
\text { villageoises }\end{array}$ & villages & $\begin{array}{l}\text { villages ne } \\
\text { compre- } \\
\text { nant } \\
\text { pas } \\
\text { d'habitants }\end{array}$ \\
\hline \hline $\begin{array}{l}\text { République } \\
\text { Sakha } \\
\text { (lakoutie) }\end{array}$ & 3103,2 & 33 & 13 & 55 & 364 & 590 & 39 \\
\hline
\end{tabular}

Tableau 2. Structure administrative de la lakoutie selon le recensement de 2002 de la Fédération russe.

Importance des villes par rapport aux villages et à la nature

Les villes sont des espaces créés essentiellement au cours de la période soviétique pour les besoins de l'industrie extractive. Ce sont des points attractifs qui accueillent les familles ayant assez de moyens financiers pour quitter les conditions difficiles de travail dans la nature. En effet, 79,6\% de l'industrie sont représentés par l'industrie minière. Pour comprendre la relation intime entre nature/lieu d'extraction/

ville/population, on peut comparer les cartes de la répartition de la population et des ressources naturelles. Les villes et cités minières sont situées sur les zones mêmes d'extraction. Leur économie et celle du pays dépendent de l'environnement immédiat. La Iakoutie est avant tout connue pour son or et ses diamants. Ainsi, la ville de Mirny à l'ouest a été construite sur les gisements de diamants découverts par les géologues en 1955 au milieu de la taïga. Elle est peuplée essentiellement de Russes, d'Ukrainiens et de Biélorusses, attirés par les hauts salaires des années soixante. D'une manière plus systématique, les cités minières sont davantage peuplées par les Russes que par les représentants des minorités sibériennes qui manquent de cadres. L'ouest de la Iakoutie est également une zone d'exploitation de gaz naturel. Le sud est concerné par 
l'exploitation de l'or à Aldan, de mica à Tommot et de charbon à Nerungri. Cette dernière cité est construite autour de la plus grande mine à ciel ouvert de la République. À l'est, on extrait surtout de l'or dans les cités minières de la région d'OustNéra. Enfin, le centre est voué à l'industrie de transformation, de construction, ainsi qu'à l'extraction de gaz naturel et de charbon. L'uranium est exploité, quant à lui, dans le nord et le nord-ouest. De par sa structure géographique complexe, la République comporte en outre d'importantes réserves d'antimoine, d'étain, de tungstène, de quartz, de vermiculite, de sel ainsi que d'une gemme unique, la tcharoïte.

Étant donné la richesse des ressources naturelles de cette République, on comprend que la création des agglomérations soit un legs de la période soviétique et un enjeu majeur pour les nouveaux souverains qui ne cessent de rogner sur l'environnement pour maintenir et développer l'activité industrielle. La Iakoutie a dû par conséquent mettre en place une politique écologique, parallèlement au développement de l'industrie, qui se double parfois de la volonté d'exploiter les ressources naturelles dans un but touristique. Dans ce domaine, l'importance de plusieurs villes se fait aujourd'hui sentir. Il s'agit tout d'abord de Iakoutsk, la capitale qui fait le relais entre les autres villes de Iakoutie et les centres importants comme Moscou, Saint-Petersbourg, Khabarovsk, Novosibirsk, Irkoustk, Blagovechensk ou Vladivostok, et qui concentre plusieurs musées (Musée national, Musée de la guimbarde, Musée de la musique des peuples du nord...). Vient ensuite le village d'Oïmiakon, connu pour être le pôle du froid de l'hémisphère nord, et qui est en passe de devenir l'une des attractions touristiques les plus originales du territoire. Nous pouvons par ailleurs citer le port de Tiksi, autrefois base militaire importante sur le delta de la Léna. Aujourd'hui dépeuplée partiellement avec la fin du complexe militaro-industriel, sa région est rendue à la nature. Elle attire les biologistes et les ornithologues qui se rendent à la station biologique du parc naturel Oust-lenski pour étudier la faune et la flore. En sus de ce tourisme qui permet de présenter des informations sur l'habitat ancien des Iakoutes et autres peuples du territoire et leurs activités quotidiennes dans la nature, se développe les écomusées (Drujba pour la culture iakoute, Bakaldyn pour la culture évenke) qui permettent de découvrir la culture des autochtones dans un espace naturel comparable à celui qu'ils occupaient avant la collectivisation. mais, malgré un attrait certain, les villages n'arrivent pas à garder les touristes car, contrairement aux villes, ils sont dépourvus de structures d'hébergement. 


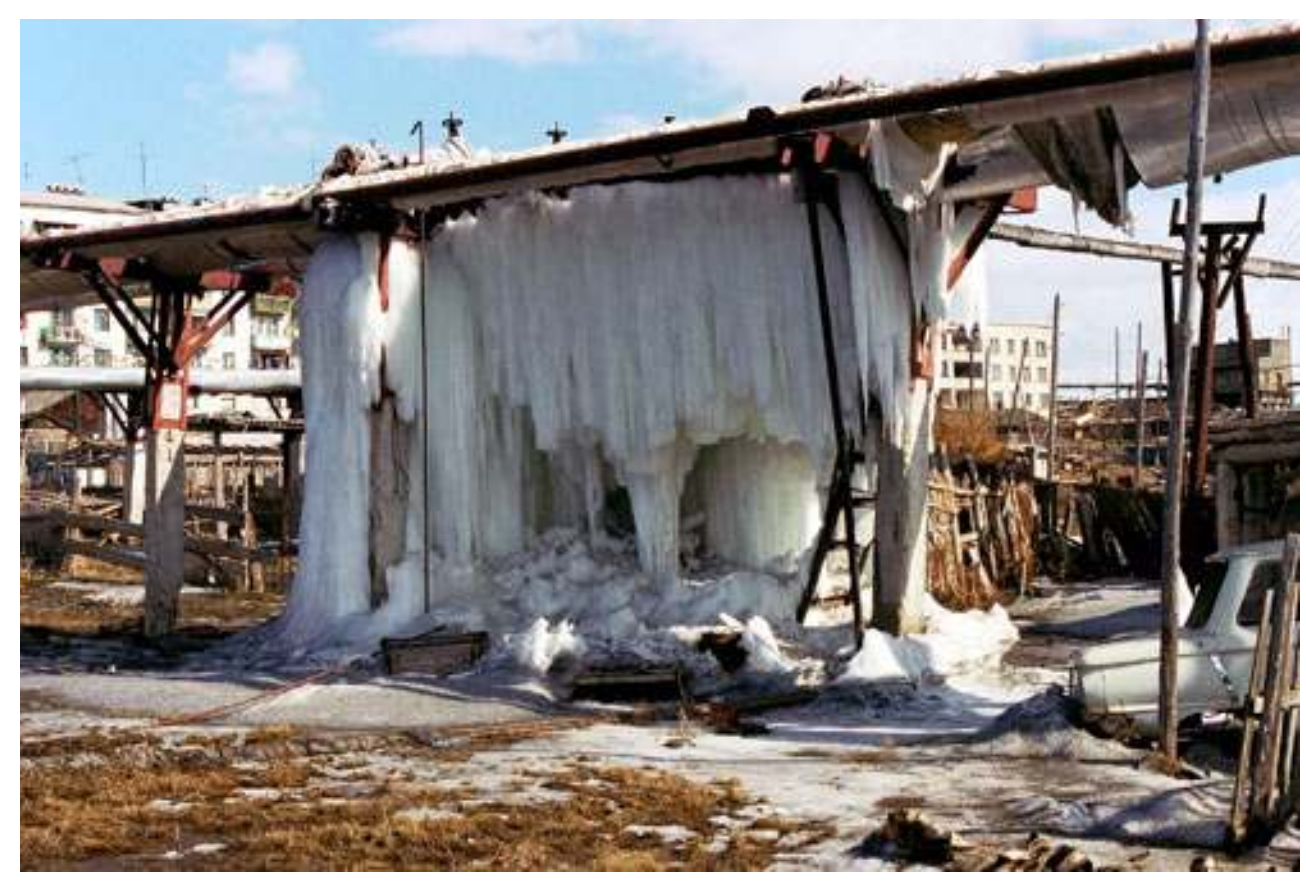

Les canalisations aériennes à lakoutsk ne résistent pas à l'hiver et se rompent, engendrant d'immenses stalactites. (C) Photo Emilie Maj, 2000.

8 Les villes restent donc le pôle d'attraction des populations rejetant les activités d'élevage non lucratives ou souhaitant envoyer leurs enfants à l'université. Quant aux agglomérations qui n'ont pas un statut ouvrier, elles se dépeuplent progressivement car celles qui possédaient des kolkhozes les ont fermés dans les années quatre-vingt-dix et la population qui n'a pas pu intégrer les nouveaux circuits de production a été contrainte de se réorienter professionnellement.

9 La ville est donc perçue par les habitants à la fois comme un espace rassurant où tout est à disposition et un espace oppressant où l'écart d'avec l'environnement naturel est mal vécu.

Le legs soviétique des cités minières : fragilisation écologique

10 La région de la Viliuï illustre bien la fragilité de la nature face aux constructions à vocation urbaine et industrielle réalisées pendant la période soviétique. La population, tout comme l'État qui met en place des plans spéciaux de réhabilitation, reconnaît que cette zone est victime de la plus grave catastrophe écologique de Iakoutie. Tout a commencé dans les années soixante avec la construction de la plus grande centrale hydroélectrique de Russie qui a nécessité l'engloutissement de milliers de $\mathrm{km}^{2}$ de forêt. Les arbres en décomposition ont libéré des phénols dans l'eau, ce qui a occasionné la mort des poissons par asphyxie. Aujourd'hui, sur 29 espèces qui peuplaient la Viliuï et son affluent la Markha, il n'en reste que $15^{7}$. On pêche dans la Viliuï en moyenne entre six à vingt fois moins de poissons que dans les autres cours d'eaux de Iakoutie. En outre, ont eu lieu dans le bassin de la Viliuï douze essais nucléaires souterrains, dont deux ont pris une tournure accidentelle. Le premier fut l'explosion de Cristal en 1974, près de la ville d'Oudatchny. D'une puissance comparable à celle d'une bombe atomique de $17 \mathrm{kt}$, elle a été cachée à la population pendant seize ans. En 1989, explosa Craton III au nord de Viliuïsk, entre Aïkhal, Oudatchny et la rivière Markha. La pollution des sols à cet endroit n'est, à présent, jamais inférieure à 70 fois la normale, autrement dit, elle est 
proche de celle qui entoure Tchernobyl. Signe d'un important déséquilibre écologique dans la région, les souris ont complètement disparu de Niurba et Sountar entre 1992 et 1993. Les conséquences sur la population sont diverses. En effet, outre les problèmes de cancers, la radioactivité provoque une fragilité des os du fait de la fixation sur le squelette du strontium radioactif, doté d'une structure identique à celle du calcium. Elle est aussi la cause d'anémies, d'une baisse de l'immunité et d'une hausse anormale du taux de mortalité. L'industrie d'exploitation de l'or et surtout des diamants, importante dans la région, rejette dans les cours d'eau des métaux lourds, des minéraux et des substances toxiques. Ainsi relève-t-on dans la région des problèmes de reins, de digestion, des anomalies congénitales, des malformations du cœur. Deux maladies sont caractéristiques de la Viliuï: la micro-élémentose, qui se caractérise par un déséquilibre des micro-éléments dans l'organisme, et "l'encéphalomyélite de la Viliuï », forme d'atteinte encéphale de la moelle épinière.

11 En réalité, le problème de l'eau est celui qui préoccupe le plus la République, à la fois dans les villages et dans les villes. On a identifié une pollution non seulement industrielle, mais agricole, due à l'intensification et à la concentration de l'élevage (bovin surtout) autour des villages. Autrefois, l'organisation sociale de l'espace (une famille par alaas) dispersait les déchets animaux sur de grandes zones. Aujourd'hui encore, les familles rassemblées dans les villages utilisent l'eau des lacs et des cours d'eau pour leur usage domestique. Or, le bétail (chevaux et vaches) boit durant tout l'hiver sur des points d'eau qui reçoivent, au moment de la fonte des glaces, une quantité trop importante d'ammoniac et de nitrate contenus par les déchets animaux. Ces substances sont responsables de problèmes de l'appareil digestif ainsi que d'hépatites. Cette maladie est fréquente chez les jeunes, même dans la capitale, dont le circuit de canalisations vétuste ne permet sans doute pas de filtrer tous les éléments pathogènes. Dans le journal Iakoutie du 27 février 2000, le Président iakoute Nikolaev faisait part de ses préoccupations à cet égard: "À l'aube du nouveau siècle, nous sommes confrontés à la nécessité d'améliorer la santé des habitants. La santé de toute la population, et plus particulièrement de la génération grandissante de notre République, devient un objet d'inquiétude. Ne pas le voir signifierait ne pas songer au patrimoine génétique des générations futures de notre pays nordique. »

La ville, élément attractif d'hommes et de capitaux

12 La capitale est aujourd'hui le lieu où se concentre importante les hommes et les capitaux. En effet, les recettes obtenues grâce à l'exploitation des ressources naturelles sont en partie consacrées à la construction de bâtiments prestigieux à Iakoutsk: nouveau théâtre, centre d'affaires, université des arts des peuples du Nord. Rares sont les subventions qu'obtiennent les gens des campagnes et c'est aussi la ville qui est chargée de redistribuer les subventions destinées aux territoires protégés sur lesquels se trouvent les villages.

13 La plupart des jeunes qui viennent étudier dans la capitale ou dans une filiale de l'université d'État à Nerungri ou Mirny retournent rarement dans leur village. Ainsi, la ville garde en son sein les cadres qui refusent d'aller travailler dans les villages, malgré la prime ajoutée à leur salaire ${ }^{8}$. Symbole de richesse et de modernité, elle est surtout peuplée de Russes. Elle n'avait pas réussi jusque-là à attirer les autochtones qui constituent encore aujourd'hui la majeure partie des habitants des villages agricoles ${ }^{9}$. Désormais, les choses changent: la ville de Iakoutsk joue un rôle d'aimant pour la jeunesse d'origine iakoute, née à la capitale, qui éprouve une aversion totale pour la 
campagne et la nature en général. Les villages du nord se dépeuplent en faveur de la capitale pour d'autres raisons: habitués à être aidés par le gouvernement sous le régime communiste, les habitants fuient les villages où l'électricité est parfois donnée par tranches horaires, par souci d'économie du diesel utilisé par les blocs électrogènes qui alimentent les villages. Les produits sont rares et à des prix bien plus élevés qu'à Iakoutsk. Ainsi, le port de Tiksi se dépeuple et près d'un quart des maisons sont à présent inhabitées. L'électricité manquant, les habitants recommencent, à la manière des anciens, à vivre au rythme de la nature dans les petites agglomérations. La pénurie de pétrole oblige les villages à vivre en économie plus fermée, comptant sur les réserves de viande fournies par les troupeaux de chevaux et de bovins et sur les productions maraîchères estivales qui se font en serres chauffées au poêle durant la nuit. Par nécessité, les habitants qui restent reviennent à un mode de vie plus proche de la nature. D'où une distance économique grandissante entre villes et villages. Dans les villages mêmes, se crée aussi une différence de niveau de vie entre les habitants qui ont des liens avec les éleveurs et les habitants qui n'en ont pas. Les premiers disposent de réserves de viande importante alors que les seconds ont davantage accès aux produits disponibles dans les magasins d'État et les nombreux petits commerces privés qui foisonnent dans les villages. Ces derniers peuvent donc vivre au village en dichotomie totale d'avec la nature, alors qu'en l'absence de ces petits magasins, c'était peu concevable.

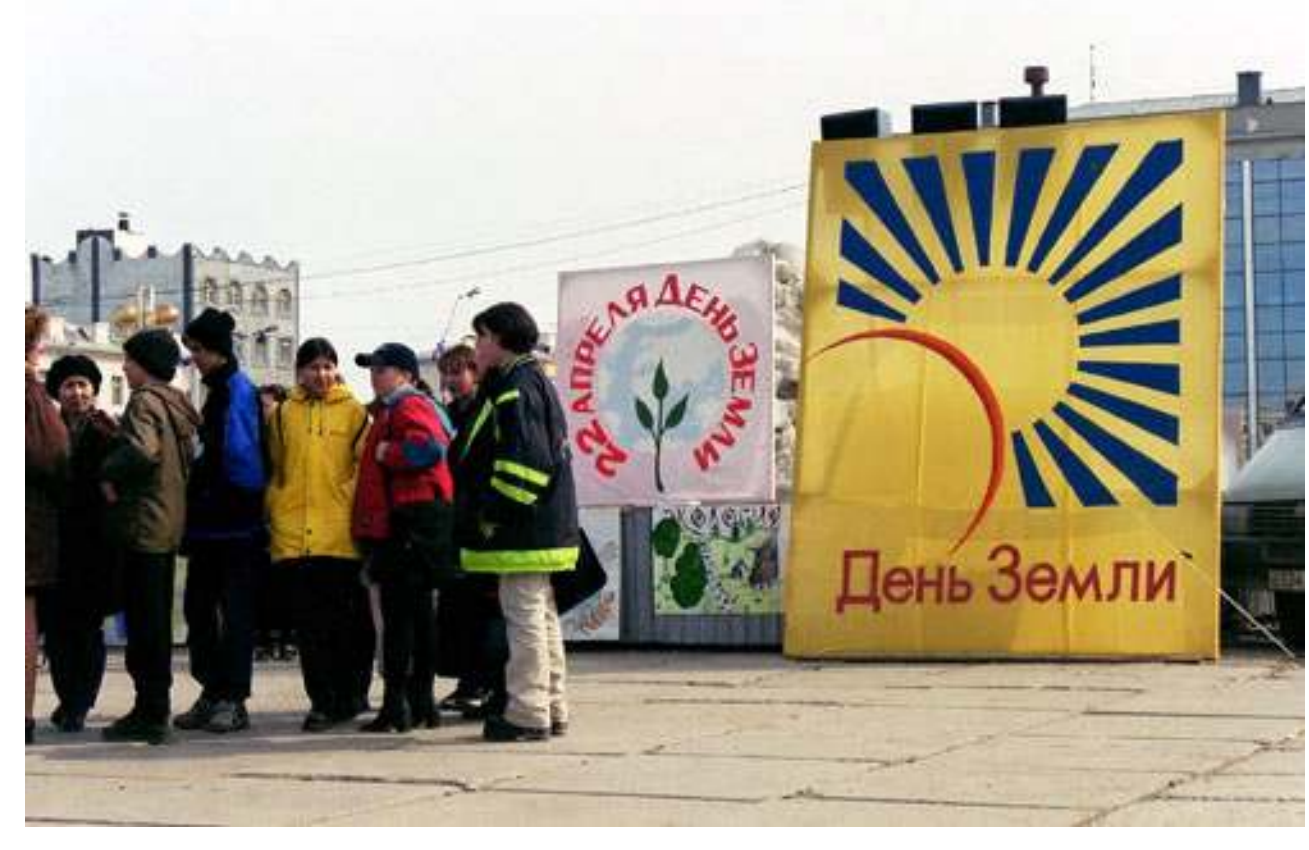

Rassemblement sur la place Lénine pour la journée de la terre le 22 avril 2000 @ Photo Emile Maj, 2000.

14 Compte tenu des difficultés économiques et des traditions, les habitants des villes qui n'ont pas accès facilement aux produits de l'élevage, de la chasse et de la pêche, se rendent chaque année dans la nature pour y ramasser baies et champignons ou constituent des réserves de viande chez des parents ou amis. Les gens qui possèdent une datch $a^{10}$ vont nouvellement y habiter tout en travaillant en ville, le temps des cultures potagères. De nombreux échanges de service interviennent aussi entre villes et 
campagne : échanges d'appartements pour les vacances, pour les étudiants. Mais ces mouvements touchent seulement les autochtones qui sont à la périphérie des villes.

La ville toujours soumise aux contraintes de la nature

Sous ce climat nordique, l'habitant des villes et celui des villages sont presque soumis à mêmes contraintes. Le citadin, pas plus que le rural, ne peut échapper aux $-45^{\circ} \mathrm{C}$, voire moins en janvier, lorsqu'il va faire ses courses ou se rend au travail. Il doit affronter le brouillard dense qui apparaît à Iakoutsk à partir de $-38^{\circ} \mathrm{C}$ et laisser le moteur de sa voiture tourner même à l'arrêt. Le facteur climatique est omniprésent dans la vie quotidienne. Il l'est également dans le domaine de la construction. Les bâtiments en béton résistent mal aux mouvements de sols qui ont lieu au moment du dégel. Il est arrivé à Iakoutsk qu'un bâtiment s'écroule en plein centre. Les canalisations se rompent fréquemment. Seules, les maisons en bois sont capables de tenir des années : on les voit se pencher, se courber, se déformer dangereusement, mais le bois suit les fluctuations de terrain et tient bon. La nature ronge la ville et celle-ci est incapable de se défendre : il faut réparer, reconstruire sans cesse ce que la nature détruit. L'Institut du Permafrost est consulté en priorité pour la construction de nouveaux bâtiments, ou même de centres d'extraction. Chargé d'étudier la structure des sols, c'est lui qui donne les directives pour la mise en place des pilotis qui constituent la fondation de toute construction en Iakoutie. Iakoutsk reste ainsi une ville de contrastes, où se côtoient immeubles de béton à sept étages et maisons en bois de plein pieds, dépourvus d'eau courante.

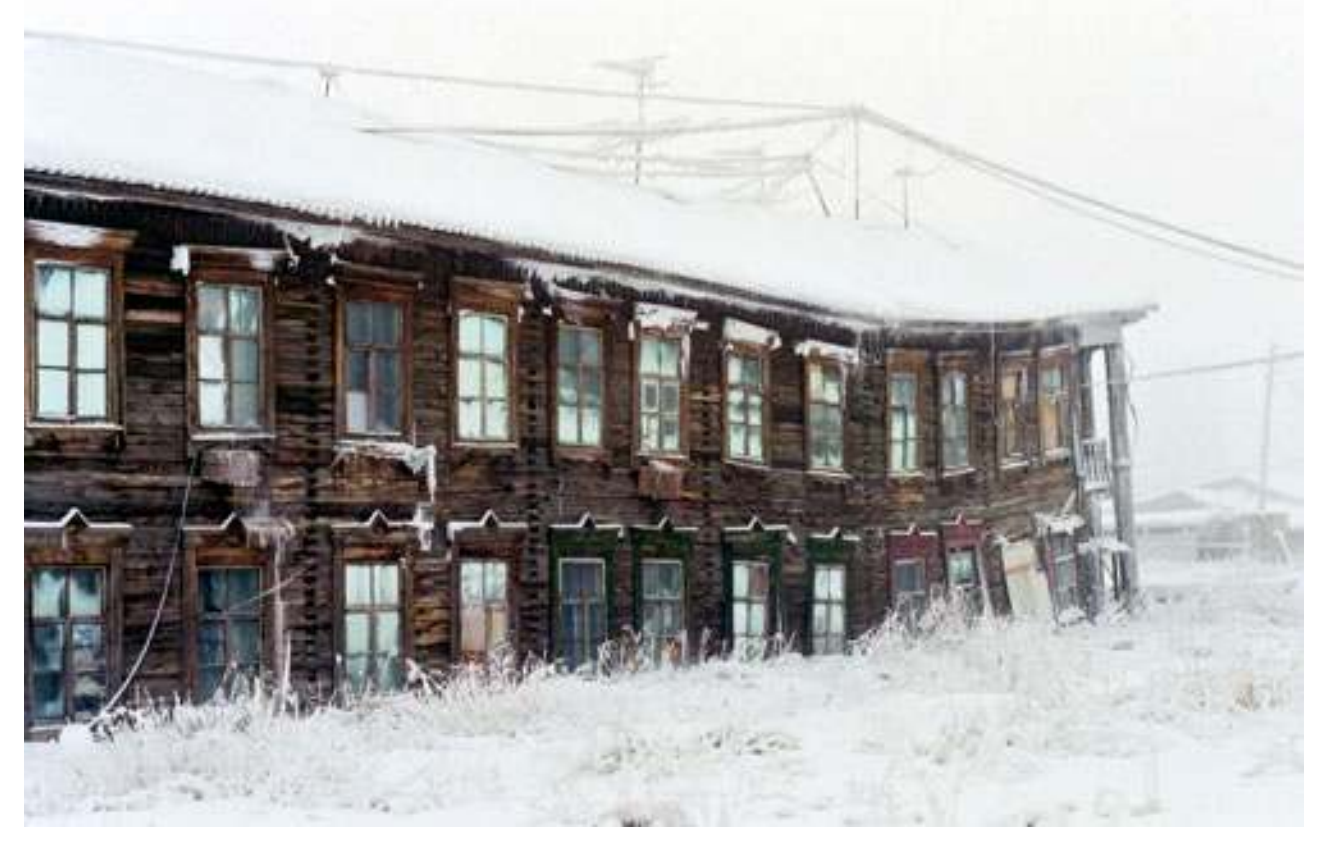

Maison abîmée par les mouvements de terrain printaniers à lakoutsk @Photo, Emilie Maj, 2000.

Chaque année la banlieue de Iakoutsk est menacée par les inondations et le fleuve Léna est bombardé pour aider l'évacuation des blocs de glace. En juin 2001, la ville entière de Lensk, près de Mirny, a été détruite et les maisons en bois retrouvées à des kilomètres plus loin, flottant aux abords d'autres villages. La nature, sans cesse, « reprend ses droits ». L'homme doit se plier aux contraintes de celles-ci. 
17 Il en est de même dans le domaine des transports. Sur la Léna, la navigation n'est possible que lorsqu'elle est dégagée des derniers blocs de glace. Aucun pont ne la traverse, en raison de la présence du permafrost et de sa largeur qui atteint par endroit $18 \mathrm{~km}$. En été, les chemins de terre deviennent impraticables. En hiver, on utilise de préférence les zimniki, routes tracées sur les cours d'eau gelés et de temps en temps balisées par quelques panneaux. Elles permettent, pour certaines d'entre elles, de se rendre jusque dans le nord éloigné, aux risques et périls du conducteur et des passagers, toujours à la merci d'une avarie, qui les condamnerait à mourir de froid ${ }^{11}$. Malgré le danger que représente le froid, il est moins facile de se déplacer en été. Les habitants sont obligés de prendre le bac, dont l'horaire n'est jamais sûr et qui est beaucoup moins rapide. Prenons l'exemple de cet entrepreneur que nous avons rencontré en 1999 et qui vendait du matériel de harnachement aux éleveurs de chevaux. Afin de commercialiser sa production, il devait faire une tournée des régions : le seul choix qui s'offrait à lui était de le faire en voiture, l'avion étant trop onéreux et le train inexistant. Mais, faire le tour d'un pays grand comme cinq fois et demie la France, seul, dans une voiture particulière sur des routes qui voient passer moins d'une dizaine de voitures par jour, n'était pas raisonnable et l'entreprise échoua, l'homme étant trop loin de ses clients potentiels...

Que ce soit donc pour la création d'un site d'extraction minière, d'un service de transports de camions ou tout simplement d'un élevage de bétail, l'entreprise en Iakoutie est souvent une affaire de personnes qui n'ont pas froid au yeux et qui acceptent de « jouer le jeu » avec la nature, de subir des pertes et de se mesurer à un partenaire sauvage et par là plein d'imprévus. Dans tous les cas, la nature enserre la ville dans un étau, la colonise et l'oblige à s'y soumettre.

Iakoutsk capitale pour la défense de l'écologie

En 1993, à Stockholm, les débats du WWF ont été alimentés par la situation écologique en Iakoutie. En avril 1995 s'est tenu le Premier Congrès républicain de protection de la nature, qui formula l'idée de mettre sous protection $25 \%$ du territoire sakha. Celui-ci aboutit au remplacement du comité socialiste soviétique de l'Écologie, créé en 1988 parallèlement au comité d'État pour l'Écologie de Moscou, par le ministère iakoute de Protection de la nature. Les écologistes de Iakoutie purent se réunir pour la première fois en 1996 lors de leur premier congrès officiel. La République signa le 28 juin 1995 avec la Fédération de Russie un accord concernant « la limitation et la transmission des pleins pouvoirs à la République dans le domaine de la défense de l'environnement et de l'exploitation de la nature ».

Grâce au concours de l'Unesco, du WWF et d'un prix gagné auprès du milliardaire Henry Sabin, la Iakoutie a réussi à donner le statut de zone protégée à $25 \%$ de sa surface totale. Iakoutsk sert ainsi d'intermédiaire avec les organisations internationales et les pays avec lesquels la République coopère, tels que le WWF, l'Unesco, le Forum du Nord, l'Institut des ressources naturelles d'Alaska et l'Allemagne ou encore le fond Sorros. La Iakoutie a ainsi reçu un prix du WWF dans le cadre du programme «WWF 2000-La planète Vive» qui prévoyait la mise sous protection d' $1 / 10^{e}$ de tout l'écosystème planétaire. Aujourd'hui, la Iakoutie est l'un des plus grands complexes de territoires protégés dans le monde. Quelques années auparavant, en 1996, la loi sur les territoires naturels protégées (ytyk kere sirder, ce qui signifie littéralement: les belles terres sacrées), définit "un ensemble d'objets et de complexes naturels, exclus en totalité ou en partie de l'utilisation industrielle et tenant compte des mœurs et des 
croyances locaux, ceci pour la protection d'un milieu naturel original, des richesses du sol et des formes traditionnelles d'agriculture ". Cette mesure répartit ces territoires selon plusieurs catégories: les fonds naturels, les parcs nationaux, les territoires naturels réservés, les réserves naturelles ainsi que les monuments naturels. Ainsi, dans le fond naturel Oust-lenski, situé sur le delta de la Léna, on a construit la station biologique la plus grande du monde, sur un territoire qui est le troisième fond protégé de la terre après l'Amazonie et le Mississipi. Ces territoires couvrent une superficie de $700000 \mathrm{~km}^{2}$.

Le renouveau de la « mentalité écologique »

En l'an 2000, l'État mit en place un programme pour "l'instruction, l'éducation et l'enseignement écologique de la population de République sakha (Iakoutie ». Dans le cadre de la mise en place de la politique écologique du pays, le ministère de la Protection de la nature tente en effet, avec la collaboration des universités et des associations, de réinculquer aux enfants le respect de la nature. Jadis l'homme du Nord ne cherchait pas à dépasser les limites que lui imposait son environnement. Bien au contraire, il ne prenait que l'indispensable, sans volonté de transformer la nature. Durant la période communiste, les Iakoutes oublièrent leur respect envers la nature au profit d'une volonté de production et de soumission des forces de la nature. Aujourd'hui, les citadins sont conscients que la vie à l'extérieur de la ville est meilleure pour la santé et envoient leurs enfants à la campagne en été. Les mérites de la viande de cheval sont vantés : "Les chevaux, à la différence des rennes, ne mangent pas de champignons et de mousses. Leur viande est dépourvue de radioactivité. » Les Iakoutes et les autres autochtones de Iakoutie sont fiers de leur environnement naturel : ils disent être les « enfants de la nature ", des "gens simples » et des " enfants de la terre de l'Olonkho », leur épopée, qui décrit richement la vie des Iakoutes sur leurs alaas.

Depuis la chute du bloc communiste, les Iakoutes essaient de retrouver ainsi leurs racines par un retour vers une harmonie avec la nature. C'est dans cette optique que le gouvernement, parallèlement à la mise sous protection du territoire, essaye de réinculquer, dans le cadre de la vie associative et de l'enseignement, les valeurs écologiques des ancêtres Sakhas. La politique écologique est mise en œuvre en corrélation avec quatre principes hérités des traditions de conservation de la nature des peuples du Nord qui se traduisent ainsi :

1. Ne cause pas de préjudice à la mère nature.

2. N'épuise pas la nature, ses bienfaits sont le patrimoine des futures générations.

3. Protège la nature, les sévices dont tu l'accables retomberont sur toi et tes descendants.

4. Aide la nature à panser les plaies que tu lui a faites. 


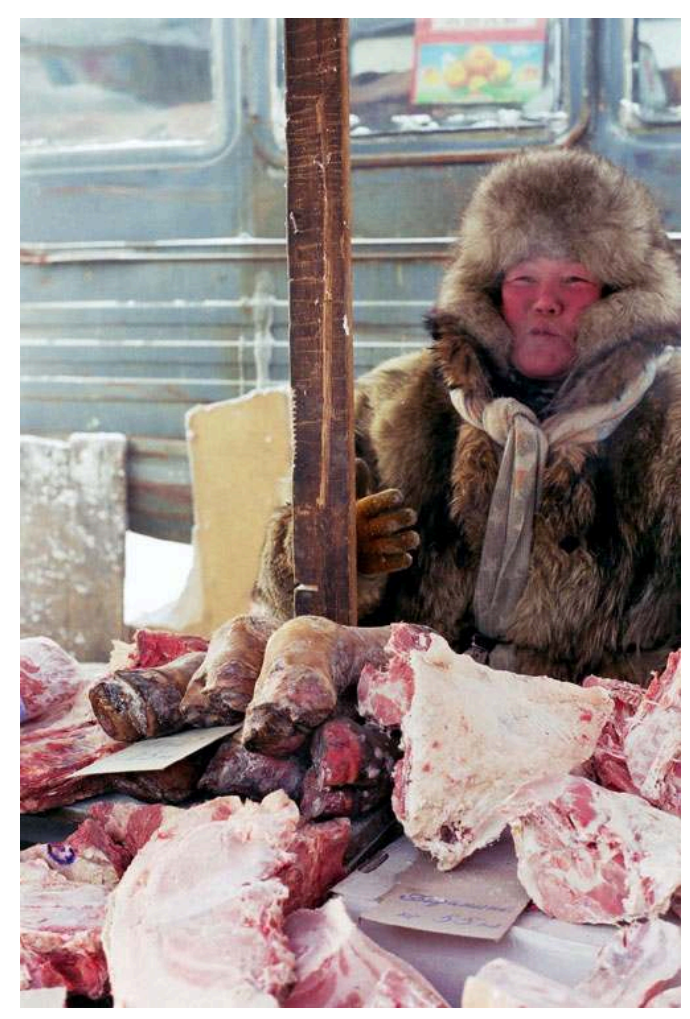

Une marchande devant son étalage de produits congelés par $-40^{\circ} \mathrm{C} \odot$ Photo Emilie Maj, lakoutsk, 2000.

Selon Valérie Alekseev, ministre de la Protection de la nature en 2000, il est indispensable «de refonder la mentalité de conquérant de la nature sur les valeurs humanistes de la personne vivant en accord, en communion avec la nature ». Ainsi, le ministère tente de réintroduire deux anciens systèmes éducatifs sakhas. Le premier est le tchyytchtchyj (littéralement: ce qui est dangereux) fondé sur l'amour de la mère et qui apprend à l'enfant le respect des autres et des animaux. Le deuxième se nomme le üeurekhe et définit les normes de comportement envers la nature lors de la chasse, de la pêche et des travaux des champs. Un nouveau chamanisme apparaît, destiné non plus seulement à soigner les hommes, mais aussi la nature en danger. Ainsi, la chamanesse iakoute Kobiakova interprète les malheurs de la population comme une vengeance de la sphère naturelle trahie par ce dernier. Pour ce faire, elle se rend dans diverses régions de la Iakoutie où la nature a souffert, afin d'y faire ses séances. L'État essaye de combler la « déspiritualisation» des villes par un grand programme baptisé «le Mode de vie sain » (en russe : Zdorovyj obraz jizni ou ZOJ) qui encourage les jeunes à faire du sport, à ne pas fumer ni boire d'alcool. Le 5 janvier a été décrété Jour de la santé et le 22 avril Jour de la Terre ou Journée de protection contre le danger écologique. Le 26 avril est pour sa part devenu le Jour du souvenir des victimes des accidents et des catastrophes nucléaires. La ville se réinvente un mode de vie sain. Les anciens chamanes disparus, l'État se charge de l'éducation au respect de la nature : devant la dégradation de la santé de la jeunesse, il voit la nécessité, en ville, de revenir à un style de vie plus «naturel». La ville se voit elle-même contrainte d'accueillir en elle la nature, mettant en place un processus d'urbanisation de la nature ou « d'ensauvagement » de la cité, pour employer un néologisme de Roberte Hamayon, qui 
va de pair avec une «dé-traditionnalisation" de la campagne et de "retraditionnalisation » de la ville.

Limites de la politique écologique

Ainsi, en Sibérie extrême-orientale, la ville n'est pas coupée de la nature, mais elle se trouve en permanente interaction avec elle, de manière que toutes deux se trouvent imbriquées l'une dans l'autre. Dorénavant, la survie de la nature dépend aussi bien de celle de la ville que l'inverse. la superficie de la République a été mis sous territoire protégé, la production minière et l'ouverture de nouveaux centres d'extraction n'en continuent pas moins. La République sakha ne peut se passer de l'industrie d'extraction qui constitue presque la totalité de ses richesses d'exportation. Son projet est de se relier aux pays du sud par un gazoduc et de développer son potentiel pétrolier. Cependant, elle doit faire face à la pression des organisations internationales comme l'Unesco et le WWF qui veillent à l'utilisation des subventions qu'elles accordent. Quoi qu'il en soit, l'écologie reste l'un des maitres mots du politiquement correct et le paradoxe a de longues années devant lui.

Durant les dix années qu'a duré sa souveraineté, seize articles de la Constitution de la Iakoutie violaient la Constitution russe, dont quatre de façon fondamentale. L'article I lui donnait le droit à l'autodétermination, l'article II à la propriété républicaine de toutes les ressources du territoire iakoute et l'article III la liberté d'établir directement des relations commerciales avec des partenaires étrangers. La situation a aujourd'hui bien changé : la République a perdu son statut de souveraineté avec V. Poutine et, se trouve, par conséquent, dépouillée du droit qu'elle s'accordait légalement sur son sol et donc de ses productions minières. Moscou devient le seul propriétaire de ses ressources, ce qui risque également de remettre en cause tous les efforts de la politique écologique mise en œuvre sous le président iakoute Nikolaev, remplacé par V. Chtyrov, russe, président de la compagnie diamantaire Alrossa (Almazy Rossij Sakha-Les diamants de Russie et de Sakha) lors des élections de décembre 2001. En outre, la Russie souhaite, à terme, redéfinir les frontières administratives de la Fédération, afin de rééquilibrer la taille des différents territoires, Républiques et autres régions y compris : il est prévu que la République sakha (Iakoutie) soit rattachée à d'autres régions, beaucoup plus pauvres qu'elle ${ }^{12}$, ce qui posera certainement des problèmes quant aux efforts menés dans le cadre de la politique écologique, notamment. Qu'adviendra-t-il du rôle joué par le ministère de la Défense de la nature si l'on prend en compte le fait que le comité d'État russe pour l'Écologie, créé en 1991, a été liquidé en 1998 sous la pression des lobbies industriels pour passer sous l'égide du ministère pro-industriel? Qu'adviendra-t-il aussi des minorités iakoutes éleveurs de chevaux ou de vaches et des autres autochtones vivant de l'élevage de renne? L'année 1998 avait été décrétée Année de l'Arctique. La Iakoutie avait décidé de la prolonger jusqu'en 2002, afin de créer une transition progressive vers une exploitation de la nature donnant priorité aux intérêts écologiques sur les intérêts économiques et d'instaurer un système de protection des minorités nordiques. Ces peuples, dont les moyens d'existence sont totalement dépendants de l'environnement, voient leur survie directement menacée par la santé déclinante de l'écosystème. L'avenir des 86 communautés nomades familiales ${ }^{13}$ de Iakoutie est rendu désormais incertain. Il faudra attendre quelques années avant de comprendre quelle seront les nouvelles orientations politiques face au contexte écologique iakoute. 
Les perspectives à l'horizon

Quels sont les perspectives de réussite de cette politique écologique de la ville vis-à-vis de la nature qui l'entoure? Dans le contexte étatique, la ville se trouve seule à décider de l'avenir des habitants de l'extérieur. Dans quelle mesure les habitants peuvent-ils retrouver une mentalité écologique directement liée à l'environnement, comme celle de leurs ancêtres, après avoir été forcés, durant la période soviétique, à une sédentarisation et une utilisation de la nature tournée vers la productivité ? Avant cela, ce que les manuels désignent sous le terme de "traditions écologiques » était constitué par un ensemble de normes, d'interdits que l'homme était contraint de respecter pour pouvoir survivre. Quel avenir peuvent par conséquent avoir ces valeurs de conservation de la nature et d'équilibre entre l'activité humaine et l'environnement pour des hommes dont l'activité quotidienne se trouve coupée de celui-ci ? Le système symbolique animiste relié à la nature et aux animaux se modifie et se codifie dans des mouvements prêts à édicter les règles religieuses à appliquer. Le nouveau chamanisme urbain fait sourire les gens de la campagne pour lesquels un chamanisme en dehors de la nature n'est pas concevable car il empêche la relation avec les esprits animaux. Une partie seulement de la population dont l'activité se rapproche de celle que menait la population iakoute dans son entier avant la période soviétique (éleveurs, pêcheurs, chasseurs) continue de comprendre les modalités de la vie en harmonie avec la nature. Dans le contexte de mondialisation et d'économie de marché actuel, l'élevage de renne, la chasse et la pêche ne permettent plus aux familles des peuples du Nord de rester au niveau des exigences des nouveaux modes de vie. Malgré la mise sous protection d'un quart de la superficie de la République avec le droit presque exclusif des représentants des minorités nordiques (communautés familiales) de jouissance de ces terres, les pronostics restent pour le moins peu encourageants si l'État n'agit pas davantage pour la conservation des activités traditionnelles des autochtones de Iakoutie, desquelles dépendent en grande partie les traditions culturelles et les valeurs. L'avenir dira ce qu'il peut advenir des politiques nationales après la reformation d'une nouvelle carte des régions de Russie par V. Poutine. Quoi qu'il en soit, le fossé se creuse de plus en plus entre la nature et la ville, qui tend à devenir un espace domestiqué où l'emprise de l'environnement est, malgré les difficultés, de plus en plus maîtrisé.

Article rédigé en 2003

\section{BIBLIOGRAPHIE}

Académie des Sciences de Russie, 1994, Ekologo-ekonomitsheskie problemy promyshlennogo osvoenija severa, Jakutsk.

Arctique Horizon 2000, 1991, Les peuples chasseurs et éleveurs, $2^{\mathrm{e}}$ colloque bilatéral francosoviétique, CNRS, Paris.

Chichlo B., 1985, Sibérie I, questions sibériennes : économie, écologie, stratégie, Paris, laboratoire de slavistique, EHESS. 
Comité gouvernemental de la Fédération de Russie pour l'Éducation supérieure, 1993, Ekologitsheskaja programma EKORS, Jakutsk.

Comité socio-écologique Viljouj, 1997, Bol' i tragedija sedogoVijouja, Iakoutsk.

Département d'Études Sibériennes, 1987, Krasnaja kniga Jakutskoj ACCP, Nauka, Novosibirsk. Gouvernement de la République sakha (Iakoutie), 1998, Gozhoudarstvenyj doklad o sostojanii okruzhajushej prirodnoj sredy Respubliki Saxa v 1997 godu, Jakutsk.

Maj É., 2002, Écologie et société en Sibérie Extrême-Orientale, l'exemple de la République sakha (Iakoutie), mémoire de maîtrise de russe, Département de langue slave de l'Université Marc Bloch (Strasbourg).

Ministère de l'Écologie, 1993, Ekologitsheskie tradicii arborigenov severa $v$ interesakh vyzhivania tshelovetshestva, Jakutsk.

Ministère de l'Éducation de la République sakha, 1993, Nacionalnaja shkola : Koncepcija i tekhnologija razvitija, Moscou (Compte-rendu de la conférence internationale du 16-21 mars à Jakutsk).

Ministère de la Protection de l'environnement de la République sakha (Iakoutie) et WWF, 2000, Osobo Okhranjaemye Prirodnye territorii (OOPT), Moscou, brochure.

Ministère de Protection de la nature de la République Sakha, 1996, Ekologitsheskie problemy Jakutii, Jakutsk.

Ministère de la Protection de la nature de la République sakha, 1997, Ekologitsheskie problemy Iakutii.

Nikolaev A. A., 2000, Osobo okhranjaemye prirodnye territorii, Jakutsk.

Nikolaeva S. N., 1993, Kak priobchtchit' rebenka k prirode, metodisheskij material djla raboty s roditeljami $v$ doschkolnykh utrezhdenijakh, Moscou.

« Okruzhajushtchaja sreda i zdorov'e arktiki », 2002, Ilken - Pec. gazeta malotshislennykh narodov severa $R S(J a), 08$ septembre, $n^{\circ} 32$, Jakutsk.

Radvanyi J., " Sibérie, l'enjeu du grenier à matières premières ", 1995, Problèmes économiques et sociaux, La Documentation Française, Paris, mai, $n^{\circ} 78$, p. 19-23.

Shmyganovski V., 1999, «Grand Nord Russe : la fin d'un rêve », Courrier International, n 433, 18 février, extrait de Izvestija.

Solomonov N. G., 1987, Besedy ob okhrane prirody severa, Jakutsk.

Thibaudat J.-P., 2000, « La Russie liquide le comité d'État pour l'Écologie », Libération, Paris, 22 juin.

Tolstikhin O. N., 1990, Okhrana prirody. Vvedenie v ingenernuju geoekologiju, Jakutsk.

Tolstikhin Iou. N., 1998, Ekologitsheskij menedgment, Novosibirsk, Nauka.

\section{ANNEXES}

\section{Géographie de la République sakha}

La Iakoutie constitue la plus grande partie de la zone nord-orientale du continent eurasiatique, avec le Kamtchatka, la Tchoukotka et la province de Magadan. Elle présente une superficie égale à $1 / 5^{\mathrm{e}}$ de la Russie, ce qui équivaut aux $2 / 3$ de l'Europe ou 
encore à 5,5 fois la France. Elle s'étend du nord au sud sur plus de $2000 \mathrm{~km}$ et d'est en ouest sur $2500 \mathrm{~km}$, soit sur trois fuseaux horaires. Plus de la moitié de son territoire se situe en zone de grand Nord et un peu moins en zone polaire. La superficie entière de la Iakoutie se trouve sur le permafrost*, dont la couche est plus ou moins épaisse du nord au sud ou en zone de montagne : au sud, il est épais de trois cents mètres et de sept cents mètres au nord, avec un pic de mille cinq cents mètres dans les montagnes de Iakoutie centrale. La Iakoutie appartient à deux plaques terrestres : la plaque sibérienne constituée de plateaux et la plaque dite verkhoïano-tchouktche aux reliefs montagneux. Les montagnes représentent $58 \%$ du territoire. Les lacs et les étangs abondent sur la première plaque, tandis que les reliefs montagneux de la deuxième privilégient les cours d'eaux qui coulent dans les vallées. Les habitants disent qu'il y a autant de lacs sur leur terre que d'étoiles brillant dans le ciel. Le pays possède plus de 443000 cours d'eau, parmi lesquels 314 mesurent plus de $100 \mathrm{~km}$. Les grands fleuves sont la Iana, l'Indigirka, l'Olenek, la Kolyma et la Léna. Ce dernier est de loin le plus important. D'une longueur de $4400 \mathrm{~km}$, c'est le deuxième fleuve de Russie par son débit. Son delta, qui occupe la superficie de $30000 \mathrm{~km}^{2}$, est le deuxième delta du monde après celui du Mississipi. La Léna est un fleuve sauvage qu'aucun pont ne traverse. Les points d'eau, eux, sont au nombre de 672 000. La Iakoutie est couverte par la toundra, la taïga et la toundra mêlée de forêt. La végétation est spécifique : du fait de l'abondance de points d'eau et de zones marécageuses, $48 \%$ des végétaux sont des plantes aquatiques et presque $20 \%$ sont constitués par les mousses, les lichens et les champignons. C'est dans un espace de taïga et de toundra que les villes s'intègrent, causant un rapport de force plus ou moins prononcé entre ville et nature d'une part, et habitants éleveurs et villageois d'autre part.

* Le permafrost aurait été découvert en 1844 par un commerçant qui voulut creuser un puits et s'aperçut que le sol était dur comme du béton. Il creusa ainsi jusqu'à une profondeur de $116 \mathrm{~m}$ et fit part de sa découverte à A. F. von Middendorf qui publia un rapport au Congrès géologique de Stockholm en 1862. Cette publication constitue en quelque sorte l'acte de naissance de la notion de permafrost. Celui-ci est aujourd'hui étudié à l'Institut de recherches sur le permafrost, fondé à Iakoutsk en 1960.

\section{La loi sur la protection de la nature en République sakha (Yakoutie)}

La loi sur la protection de la nature en République sakha (Yakoutie) oblige chaque citoyen (art.1) :

- À garantir la préservation de la nature

- À prévenir toute influence écologiquement négative de l'activité humaine sur la nature

Elle déclare le droit à tous les citoyens de la république à :

- Un habitat écologiquement favorable

- Un régime d'utilisation de la nature, capable de garantir les intérêts des générations futures

Elle désigne comme prioritaires (art. 2) :

- Les problèmes de défense de la vie et de la santé de l'homme sur les autres problèmes écologiques 
- Les problèmes écologiques sur les problèmes économiques dans leur combinaison rationnelle, garantissant à l'homme le droit à un milieu de vie favorable

- Les programmes de protection de la nature, prescrivant une exploitation rationnelle de la nature, sur les autres programmesElle prescrit (art. 3) de :

- Établir des priorités pour les programmes écologiques, en les divisant en protection contre la pollution, les feux de forêt, les détériorations, l'épuisement des ressources et l'exploitation non rationnelle de la nature

La loi constate (art. 4) que le territoire de la république est une zone à laquelle on peut facilement causer des dégâts, et qui a une capacité écologique limitée. C'est pourquoi la loi interdit :

- L'élaboration et la réalisation de projets, liés au dépassement de l'équilibre des complexes écologiques et à la destruction de l'écosystème

En République sakha (art. 5) :

- On donne la priorité aux technologies écologiquement pures et sans rejet

- On crée des territoires et objets spécialement protégés

Les plus grandes limitations sont (art. 29) :

- L'activité économique du gouvernement ne doit pas porter préjudice à la nature

- On doit établir un contrôle efficace de l'état de la nature en se basant sur les critères et paramètres internationaux

La loi sur la protection de la nature désigne comme indispensable (art. 6) :

- L'institution d'une politique écologique unique dans la république

Le Président de la République a formulé les principes de politique écologique suivants :

- L'intégration réciproque de l'écologie et de l'économie indépendamment du régime politique, de la conception du monde et de la mentalité du gouvernement et du peuple. En atteignant une telle fusion et une telle intégration, l'économie et l'écologie, leur conflictualité inhérente, leur égoïsme, leurs différences, se présentent comme un système unique

- La priorité de la défense du patrimoine génétique de l'homme et de l'environnement lors des différents scénarios de développement de la production et de l'économie en accord avec la capacité écologique et l'équilibre écologique des régions, des provinces et des zones

- Le bien-fondé scientifique du caractère payant de l'exploitation de la nature au cours de la limitation rigoureuse des intérêts économiques, des besoins des générations présentes et futures en accord avec les traditions écologiques séculaires, la culture écologique, la spiritualité des peuples, des régions peuplées par les autochtones

- La garantie législative et constitutionnelle de la survie, de la conservation du développement balancé de la nature, de l'indemnisation des dégâts écologiques et des travaux de rétablissement de la nature, dans le respect du droit de l'homme à un lieu d'habitation sain et à la sécurité écologique 
- La fusion des niveaux régionaux, fédéraux et internationaux de résolution des problèmes écologiques, car, quel qu'il soit, un problème écologique naissant dépasse les frontières de chaque région, du pays, et exige la consolidation des efforts de la communauté des pays et des régions

- La mise sous réserve pour les futures générations de $20 \%$ du territoire de la région sous le régime d'une protection spéciale

\section{NOTES}

1. Pour plus de commodités, nous garderons dans notre article une transcription française, voire francisée, des noms (propres et communs) iakoutes et russes.

2. L'ajout de l'épithète « sakha » exprime à lui seul la volonté de singularisation du peuple iakoute ainsi que l'identification, ou plutôt la recherche de leurs origines, au sein de l'ensemble des peuples d'Asie centrale. Cet épithète est surtout usité dans les épopées. En effet, Urjangkhaï Sakhalar est le nom mythique des Iakoutes que citent leurs épopées Olonkho. Sur le blason de la République sakha figure un pétroglyphe de la région du Baïkal représentant un cavalier avec un drapeau.

3. Les produits de cette mine célèbre étaient reversés en partie à la compagnie sudafricaine De Beers. Le statut des diamants de Mirny a changé depuis deux ans.

4. Verkhoïansk et Oïmiakon sont reconnus pour être les pôles du froid de l'hémisphère Nord, avec, à Oïmiakon, une température record de $-71,8^{\circ} \mathrm{C}$, à laquelle on a dédié un monument.

5. L'alaas était jadis le lieu de vie des Iakoutes qui établissaient leur maison/yourte/ balagan, et leurs troupeaux sur une prairie entourée de forêts, bordée de collines et ancrée autour d'un point d'eau (cours d'eau ou lac).

6. Oulous : division administrative correspondant à la région.

7. Les données chiffrées et concernant les problèmes écologiques et de santé sont toutes extraites du rapport annuel gouvernemental de la situation écologique en Iakoutie pour les années 1996 et 1977.

8. Il existe en Russie un système de prime accordée en fonction d'un « coefficient de septentionalité » chiffré entre 0 et 1000 points suivant un critère précis (climat, végétation, accès aux transports). La Terre François-Joseph a un coefficient de 875, l'île Wrangel de 800..., Iakoutsk de 300 .

9. $60 \%$ de la population iakoute vit en dehors des villes et $14,5 \%$ de la population active travaille dans l'agriculture.

10. Mot russe pour désigner la maison de campagne dotée d'un petit jardin comprenant une serre où le propriétaire plante généralement tomates, concombres, carottes, betteraves et choux.

11. Pour cette raison, les convois qui se rendent dans les régions nordiques sont toujours composés d'au moins deux véhicules, afin de protéger le conducteur d'une panne qui le condamnerait à mourir gelé.

12. Elle ne sera pas, par exemple, rattachée à la Tchoukotka qui comporte le port de pêche le plus important de Russie, ce qui créerait une région forte économiquement avec non seulement les réserves minières de Iakoutie mais les ressources maritimes de la Tchoukotka. 
13. Les communautés nomades familiales avaient été démantelées par le pouvoir soviétique. On les a vues renaître après la chute de l'URSS. En Iakoutie, une loi sur ces communautés a été adoptée en janvier 1993 par la 16e session de l'Assemblée d'Etat de la République Sakha (Iakoutie). Au $1^{\text {er }}$ mars 1993, on recensait sur le territoire de la République au sein de ces communautés 7202 membres, dont 3889 membres actifs.

\section{RÉSUMÉS}

Durant les années soviétiques, l'homme a entrepris de dominer la nature vierge de l'est de l'Oural. Sédentarisant des populations nomades ou semi-nomades qui vivaient dispersées sur de grandes étendues pour y construire des villes minières en particulier, l'URSS a causé des dommages à l'environnement dans les années soixante qui se ressentent aujourd'hui. Dans la partie orientale de la Sibérie, la création des villes remonte au début du $\mathrm{xx}^{\mathrm{e}}$ siècle Pour comprendre les enjeux d'une politique écologique dans le contexte sibérien, on se posera ici les questions suivantes : qu'est-ce qu'une ville de Sibérie dans un pays de taïga et de toundra qui vit essentiellement de l'industrie minière dans le contexte post-communiste ? Comment l'espace citadin s'inscrit-il dans l'environnement? Quelles relations entretient-il à la nature? Pour répondre à ces questions, nous décrirons tout d'abord l'espace environnemental de la nature, des campagnes et des villes. Puis, nous nous demanderons comment ces nouvelles républiques autonomes de Sibérie comme la Yakoutie gèrent l'héritage soviétique, prises entre le désir de mettre en œuvre une politique écologique sous les sollicitations à la protection de l'environnement des organisations internationales et les nécessités de survie économique qui dans le contexte russe et mondial les incitent à exploiter à nouveau des richesses minières au mépris de leurs engagements en faveur de la protection environnementale. La république Sakha (Iakoutie) servira de cadre à notre recherche.

Emilie Maj. Soviet legacy and environmenal stakes in Siberian city

During the Soviet years, man tried to master virgin nature East of the Urals. By settling nomadic or semi-nomadic populations living on vast tracts of land to build mining towns in particular, the USSR caused environmental damages in the 1960's which are now felt today. To understand what is at stake in an ecological policy in the Siberian context, the following questions will be asked: what is a Siberian town in a Taiga and Toundra country, that derives most of its living from mining industry in the post-communist context? How does the urban space fit in the environment? What relationship does it have with nature? To answer these questions, we will first describe the environmental space of nature, countryside and town. Then, basing our research on the Sakha republic of Yakutia, we will ask how the new autonomous Siberian republics manage the Soviet heritage, as they are caught between the desire to put in place an ecological policy to protect the environment in response to appeals by international organisations, and the necessities of economic survival which incite them to start exploiting their mining wealth again in spite of the environmental protection promises they have made. 
AUTEUR

ÉMILIE MAJ

Doctorante, EPHE associée au Groupe Sociétés, Religions, Laïcités (GSRL), emiliemaj@hotmail.com 